Article

\title{
The Structural Characteristics and Biological Activities of Intracellular Polysaccharide Derived from Mutagenic Sanghuangporous sanghuang Strain
}

\author{
Tingting $\mathrm{Li}^{1,2,+}$, Linjun Chen ${ }^{2,+}{ }^{+}$, Di Wu ${ }^{3}$, Guochao Dong ${ }^{2}$, Wanchao Chen ${ }^{3}$, Henan Zhang ${ }^{3}$, \\ Yan Yang ${ }^{3, *}$ and Wenhui $\mathrm{Wu}^{1, *(\mathbb{D})}$ \\ 1 College of Food Science \& Engineering, Shanghai Ocean University, Shanghai 201306, China; \\ litt@sumhs.edu.cn \\ 2 College of Medical Technology, Shanghai University of Medicine \& Health Sciences, Shanghai 201318, China; \\ chenlj@sumhs.edu.cn (L.C.); dongguochao66@163.com (G.D.) \\ 3 College of Medical Technology, Shanghai Academy of Agricultural Sciences, Shanghai 201403, China; \\ wudi@saas.sh.cn (D.W.); chenwanchao@saas.sh.cn (W.C.); henanhaoyun@126.com (H.Z.) \\ * Correspondence: yangyan@saas.sh.cn (Y.Y.); whwu@shou.edu.cn (W.W.); \\ Tel.: +86-21-6220-9765 (Y.Y.); +86-21-6190-0388 (W.W.) \\ + These authors contributed equally to this work.
}

Received: 21 July 2020; Accepted: 10 August 2020; Published: 13 August 2020

\begin{abstract}
Sanghuangporous sanghuang is a rare medicinal fungus which contains polysaccharide as the main active substance and was used to treat gynecological diseases in ancient China. The intracellular polysaccharide yield of $S$. sanghuang was enhanced by the strain A130 which was screened from mutant strains via atmospheric and room temperature plasma (ARTP) mutagenesis. The objective of this research was to investigate the effects of ARTP mutagenesis on structural characteristics and biological activities of intracellular polysaccharides from S. sanghuang. Six intracellular polysaccharide components were obtained from S. sanghuang mycelia cultivated by the mutagenic strain (A130) and original strain (SH1), respectively. The results revealed that the yields of polysaccharide fractions A130-20, A130-50 and A130-70 isolated from the mutagenic strain fermentation mycelia were significantly higher than those of the original ones by 1.5-, 1.3- and 1.2-fold, and the clear physicochemical differences were found in polysaccharide fractions precipitated by $20 \%$ ethanol. A130-20 showed a relatively expanded branching chain with higher molecular weight and better in vitro macrophage activation activities and the IL-6, IL-1, and TNF- $\alpha$ production activities of macrophages were improved by stimulation of A130-20 from the mutagenic strain. This study demonstrates that ARTP is a novel and powerful tool to breed a high polysaccharide yield strain of S. sanghuang and may, therefore, contribute to the large-scale utilization of rare medicinal fungi.
\end{abstract}

Keywords: polysaccharides; structural characteristics; biological activities

\section{Introduction}

Sanghuangporous sanghuang, a traditional Chinese medicinal fungus, belongs to Basidiomycota, Hymenomycetes, Aphyllophorales, Hymenochaetaceae and Sanghuangporus, and usually grows on mulberry, birch and other Morus L. (Morus alba Linn) plants in the regions of central and southern China [1,2]. It has been reported to possess effective anti-cancer, anti-inflammatory, anti-oxidative and stimulating immunity properties [3-5]. Polysaccharides are the main bioactive macromolecules of S. sanghuang, which provide a series of immunological functions [6]. The prevalence rates of cancer and chronic metabolic diseases are on the rise, and S. sanghuang is a prospective natural resource for the development of functional food and drugs with high safety. 
High-quality strain is the important factor to obtain $S$. sanghuang products with high polysaccharide content. However, in the last few decades, much attention was focused on fermentation, isolation and biological activities of $S$. sanghuang, and there were few studies focused on strain breeding. ARTP mutagenesis is a novel microbial mutation breeding technology driven by radio-frequency power, affecting the structure and permeability of the cell wall and plasma membrane, followed by DNA damage, including missense mutation, deletion or frame shift mutation [7-9]. This technique can effectively mutate bacteria, microalgae, fungi, yeast, and actinomycetes, etc. [10-12]. Wen Jiang [13] screened and bred Monascus on the production of extracellular polysaccharides by UV-ARTP composite mutagenesis, and the polysaccharide production of mutant was $61.18 \%$ higher than that of parent strain. Fang et al. [14] reported that the ARTP could cause diverse mutations of the microalgae Spirulina platensis and increase the polysaccharide content by 1.8-fold.

Subsequently, ARTP mutation has been successfully applied to improve the polysaccharide production of edible fungi. Wei et al. [15] mutated a Ganoderma lucidum strain by ARTP and found that the mycelia yield increased by $25.03 \%$ and its polysaccharide content increased by $44.66 \%$. Zhu et al. [16] reported that the structural characteristics of polysaccharide from the mutant $H$. erinaceus strain were significantly different from original strain and the in vitro immune activity was enhanced. In our previous studies, a new S. sanghuang strain A130 with high polysaccharide content was bred through ARTP mutagenesis after screening and a genetic stability test, which showed a productivity increase of polysaccharides and a total productivity of biomass compared with those of the original strain SH1. What are the differences between the polysaccharides produced by this new S. sanghuang strain A130 and the original one in the structure and activity and how does ARTP affect the production of polysaccharides need to be further investigated. The chemical composition, molecular mass and chain conformation of polysaccharides are essential for the study of their bioactivities. It is a rather comprehensive task to reveal the changes in physicochemical properties and biological effects of high-polysaccharide-yield strain A130 after mutation. Therefore, in this study, the physicochemical properties and immunological activities of S. sanghuang polysaccharides between A130 and SH1 are systematically compared and the change of conformation of polysaccharides is studied by high performance size-exclusion chromatography (HPSEC) chromatography combined with laser light scattering and viscometry. The study attempts to unravel the interaction relationship between structure and activity, which enables further exploration of the mechanism of high polysaccharide productivities by ARTP.

\section{Results and Discussion}

\subsection{Biomass and Polysaccharide Contents}

The mutant strain A130 and the original strain SH1 were cultured by liquid fermentation with the same inoculation amount, respectively. There are great differences between these two treatments and the comparison in the average biomass and polysaccharide content. According to Table 1, the average biomass of mutant strain A130 increased by $14.43 \%$ compared with the original strain SH1. Meanwhile, the total polysaccharide contents of the mutant strain A130 increased by $4.53 \%$. Therefore, the mutant strain A130 was a new strain with high polysaccharide yield and genetic stability. The high positive gene response effect, as well as unique response mechanism, contributes to the genetic stability of the mutagenesis mutant strain A130. Meanwhile, high-energy active particles from ARTP could repair the genetic damage, thus the characteristics from mutant strains could be stably inherited. The characterization of polysaccharides and biological activities are very important for efficient applications, therefore, we studied the differences of polysaccharide after ARTP mutation.

\subsection{Chemical Composition of Polysaccharides}

Six polysaccharide fractions were isolated by grade $\mathrm{EtOH}$ precipitation and the monosaccharide components of their hydrolyzates were analyzed by high-performance anion exchange chromatography 
(HPAEC), and the results are summarized in Table 2, together with polysaccharide content and yields of the polysaccharide fractions. The results indicated that glucose was the predominant monosaccharide in S. sanghuang polysaccharide. The polysaccharide fractions SH1-20 and A130-20 consisted mainly of glucose and mannose, with ratios 7.3:1.0 and 15.0:1.0, respectively. The ratio of glucose in A130-20 was double of that in SH1-20, which indicated that ARTP mutagenesis increased the proportion of glucose in polysaccharides, and the anabolic pathways of polysaccharides in the mutant strain were changed. Owing to ARTP mutagenesis, the yields of mutant polysaccharide fractions A130-20, A130-50 and A130-70 were significantly higher than the original one by 1.5-, 1.3- and 1.2-fold. The polysaccharide content of A130-20, A130-50 and A130-70 from the mutated strain improved by 23, 14, and 11\% compared with original strain SH1, as shown in Table 2.

Table 1. Biomass and mycelium polysaccharide contents.

\begin{tabular}{ccccc}
\hline Strain No. & Biomass (g/L) & Increasing Rate (\%) & Polysaccharide Content (\%) & Increasing Rate (\%) \\
\hline A130 & $15.8 \pm 0.13$ & $14.43 \pm 0.12$ & $4.54 \pm 0.05$ & $19.16 \pm 0.08$ \\
SH1 & $13.52 \pm 0.05$ & 0 & $3.81 \pm 0.12$ & 0 \\
\hline
\end{tabular}

Note: All results are means of three parallel samples, and data are reported in mean \pm standard deviation.

Table 2. Monosaccharide composition, polysaccharide content, and yield of S. sanghuang polysaccharides from mycelia.

\begin{tabular}{|c|c|c|c|c|c|c|c|c|c|c|c|}
\hline \multirow{2}{*}{ Samples } & \multicolumn{9}{|c|}{ Monosaccharide Composition of Polysaccharide Fractions } & \multirow{2}{*}{ Yield (\%) } & \multirow{2}{*}{ Polysaccharide Content (\%) } \\
\hline & Fuc & Ara & GlcN & Gal & Glc & Xyl & Man & GalA & GluA & & \\
\hline SH1-20 & - & - & - & - & 7.30 & - & 1.00 & - & - & $0.40 \pm 0.15$ & $63.61 \pm 0.48$ \\
\hline A130-20 & - & - & - & - & 15.0 & - & 1.00 & - & - & $0.58 \pm 0.15$ & $78.16 \pm 0.31$ \\
\hline SH1-50 & - & - & - & 0.24 & 1.73 & - & 1.00 & - & 0.11 & $0.43 \pm 0.08$ & $56.67 \pm 0.38$ \\
\hline A130-50 & - & - & - & 0.14 & 2.30 & - & 1.00 & - & 0.13 & $0.56 \pm 0.10$ & $64.07 \pm 0.50$ \\
\hline SH1-70 & - & - & - & - & 3.40 & - & 1.00 & - & - & $0.54 \pm 0.06$ & $68.12 \pm 0.21$ \\
\hline A130-70 & - & - & - & - & 2.10 & - & 1.00 & - & - & $0.65 \pm 0.12$ & $76.72 \pm 0.40$ \\
\hline
\end{tabular}

Note: The values represents molar ration in the table; "_": not detected or very little content. " \pm ": values shown were the means \pm SD of 3 replicates; Fuc, fucose; Ara, arabinose; Xyl, xylose; Man, mannose; Gal, galactose; Glc, glucose.

A130-50 and SH1-50 had similar monosaccharide composition, which were composed of galactose, glucose, mannose, and glucuronic acid with similar composition ratios of nearly 0.2:2:1:0.1. In addition, monosaccharide compositions of mannose and glucose were 3.4:1 and 2.1:1 in SH1-70 and A130-70 respectively. The results showed that the monosaccharide composition in $20 \%$ ethanol-precipitated polysaccharide fractions were greatly changed after ATRP mutation, while there was little change in 50 and $70 \%$ ethanol-precipitated polysaccharide fractions. The results revealed that high-energy active particles from ARTP significantly affected the chemical components and the structural characteristics of polysaccharides in mycelia, especially for the polysaccharide fractions precipitated by $20 \%$ ethanol.

\subsection{Infrared Spectra Analysis of Polysaccharides}

The infrared spectra of six polysaccharide fractions are shown in Figure 1 . The characteristic IR absorption of polysaccharide at $3438 \mathrm{~cm}^{-1}(\mathrm{O}-\mathrm{H}), 2958 \mathrm{~cm}^{-1}(\mathrm{C}-\mathrm{H}), 1644 \mathrm{~cm}^{-1}$ (H-O-H bending), $1156 \mathrm{~cm}^{-1}$ (C-O), and $1076 \mathrm{~cm}^{-1}$ (C-O-C) were exhibited in all samples. The IR absorptions of A130-20 and SH1-20 at $1540 \mathrm{~cm}^{-1}$, corresponding to the secondary-CONH-group in the protein, indicating that there were proteins bonded to the polysaccharides in $20 \%$ ethanol-precipitated polysaccharide fractions. SH1-20 showed characteristic absorption of an $\alpha$-D-glucan IR at $850 \mathrm{~cm}^{-1}$, while SH1-20 showed clear IR absorption at $930 \mathrm{~cm}^{-1}$, revealing the existence of $\beta$-D-glucan. The IR spectra of A130-50 and SH1-50 are almost identical to each other, indicating that they have the similar chemical structure, and that they are mainly different in molecular weight. Furthermore, the samples SH1-50 and A130-50 showed peaks at $1720 \mathrm{~cm}^{-1}$ (COO-), indicating the presence of glucuronic acid, which was consistent with the result obtained by HPAEC determination. A130-70 and SH1-70 showed similar IR absorption and characteristic absorptions of polysaccharide, the weak bands at $2958 \mathrm{~cm}^{-1}$ were ascribed to C-H stretching, while the 
bands at $1416 \mathrm{~cm}^{-1}$ were attributed to the $\mathrm{C}-\mathrm{H}$ bending vibrations. The results of IR spectra showed ARTP mutation did not change the spectral characteristics of the $S$. sanghuang polysaccharides.

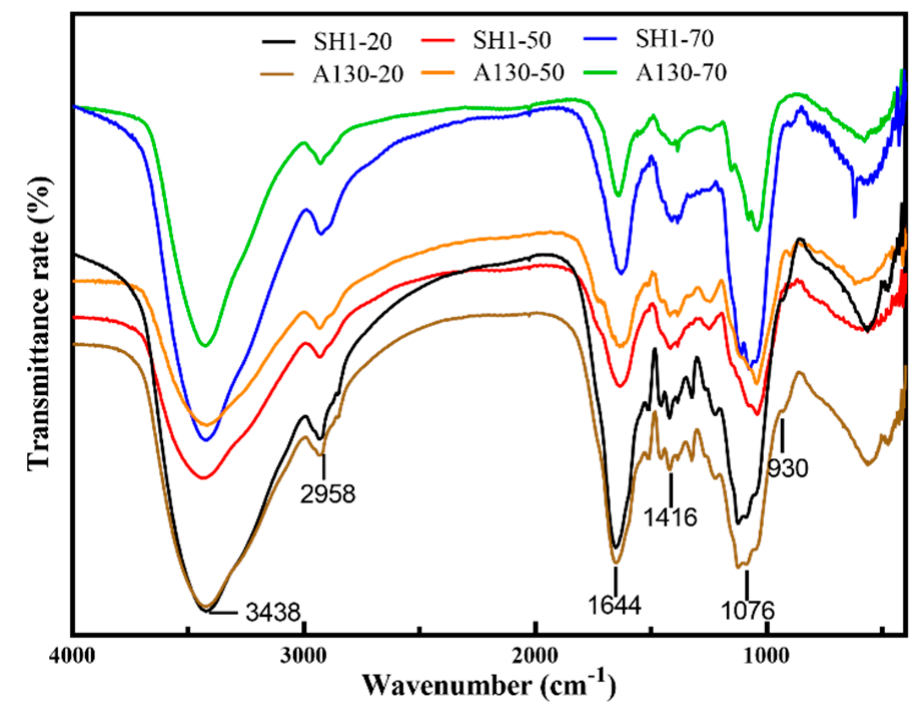

Figure 1. The infrared spectra of six polysaccharide fractions.

\subsection{The Effects of Mutation on Molecular Weight Distribution}

HPSEC chromatograms of the six fractions are shown in Figure 2 and the values of weight-average molecular weight $(M w)$, number-average molecular weight $(M n)$ and polydispersity $(M w / M n)$, root mean square radius (rms radius) and intrinsic viscosity $[\eta]$ values by HPSEC-MALLS and viscometry measurements combined with laser light scattering photometry and a differential refractive index detector were listed in Table 3. A130-20 exhibited two polysaccharide peaks, while SH1-20 showed only one single peak. The molecular weight $(\mathrm{Mw})$ of A130-20 (peak1) attained to $1.588 \times 10^{7} \mathrm{Da}$ and $\mathrm{Mw}$ of peak2 was about $8.045 \times 10^{6} \mathrm{Da}$ which was very close to that of SH1-20 $7.839 \times 10^{6} \mathrm{Da}$. This indicated that Peak1 of A130-20 was the new polysaccharide fraction induced by ARTP mutagenesis and characterized by a large molecular weight. The HPSEC chromatogram of A130-50 was similar to that of SH1-50, and two peaks which appear closest to each other were identified. The weight-average molecular weights of A130-50 and SH1-50 ranged from 3.520 $\times 10^{5}$ to $1.336 \times 10^{6} \mathrm{Da}, 1.875 \times 10^{5}$ to $1.326 \times 10^{6} \mathrm{Da}$, respectively. There was no significant difference in HPSEC chromatograms of A130-70 and SH1-70, which showed a narrow peak with low molecular weight of $2.506 \times 10^{4}$ and $2.325 \times 10^{4} \mathrm{Da}$, respectively.

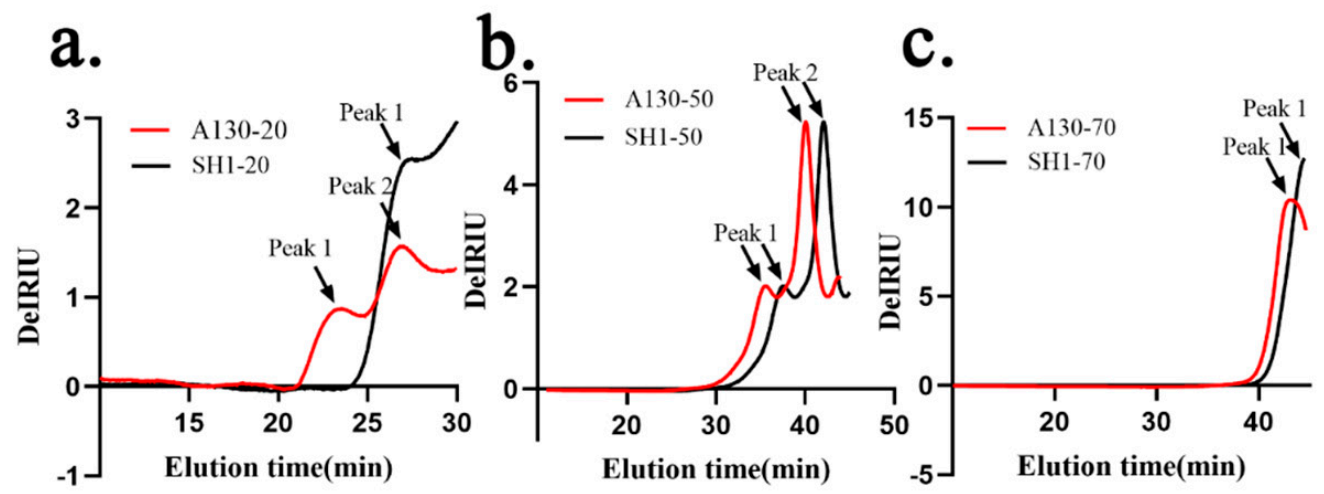

Figure 2. High performance size-exclusion chromatography (HPSEC) chromatograms of the six polysaccharide fractions. (a) HPSEC chromatograms of A130-20 and SH1-20; (b) HPSEC chromatograms of A130-50 and SH1-50; (c) HPSEC chromatograms of A130-70 and SH1-70. 
Table 3. The molecular weight distribution of $S$. sanghuang polysaccharide fractions.

\begin{tabular}{cccccccc}
\hline Fraction & Peak & Mw (Da) & Mn (Da) & rms Radius (nm) & [ ] Values (mL/g) & Polydispersity (Mw/Mn) & Percentage (\%) \\
\hline \multirow{2}{*}{ A130-20 } & Peak1 & $1.588 \times 10^{7}$ & $1.367 \times 10^{7}$ & 494 & 1020.50 & 1.162 \\
SH1-20 & Peak2 & $8.045 \times 10^{6}$ & $7.327 \times 10^{6}$ & 48.5 & 560.20 & 1.099 \\
Peak1 & $7.899 \times 10^{6}$ & $9.114 \times 10^{6}$ & 64.4 & 486.5 & 1.023 \\
A130-50 & Peak1 & $1.336 \times 10^{6}$ & $1.210 \times 10^{6}$ & 47.8 & 320.10 & 1.104 \\
& Peak2 & $3.520 \times 10^{5}$ & $3.459 \times 10^{5}$ & 38.5 & 160.85 & 1.018 \\
SH1-50 & Peak1 & $1.326 \times 10^{6}$ & $1.210 \times 10^{6}$ & 46.8 & 310.65 & 1.096 \\
A130-70 & Peak2 & $1.875 \times 10^{5}$ & $1.759 \times 10^{5}$ & 40.2 & 130.34 & 1.067 & 36.5 \\
SH1-70 & Peak1 & $2.506 \times 10^{4}$ & $2.410 \times 10^{4}$ & 38.5 & 120.40 & 1.040 & 31.3 \\
\hline
\end{tabular}

Based on the data above, we can find the weight-average molecular weight of polysaccharide fractions decreased with the increasing of alcohol precipitation concentration. The molecular weight of $20 \%$ ethanol-precipitated fraction greatly increased after ARTP mutation, but little change was found in 50 and $70 \%$ ethanol-precipitated fractions, which well coincided with the monosaccharide composition analysis. The polysaccharide (A130-20, peak1) is a totally new macromolecular polysaccharide found in mutant strain, from which we could infer ARTP mutation stress promoting the aggregation of macromolecular polysaccharides of S. sanghuang and making the changes of polysaccharide biosynthesis. As the proportion of glucose in A130-20 is double of that in SH1-20, the new polysaccharide fraction in A130 (peak1) could possibly be a kind of glucan.

\subsection{The Effects of Mutation on Chain Conformation of A130-20 and SH1-20}

An analysis of conformation plots according to molecular mass and root mean square (RMS) radius could give more structure information of polysaccharides [17]. The distributions of molar mass and rms radius are shown in Figure 3a,b respectively. A130-20 showed larger molar mass but smaller rms radius than SH1-20 at the same elution time, which indicated A130-20 was more tightly structured compared with SH1-20. The RMS conformation plot provided relations between rms radius and molar mass [18,19]. To further elucidate the structure differences between A130-20 and SH1-20, conformation plots were detected and calculated by Astra 6.1 in Figure 3c. The sever oscillation curve in RMS conformation plot of A130-20 indicates a relatively expanded branched chain such as $\alpha$-D-glucan $[20,21]$, which was in accordance with its tight structure. It is generally accepted that the slopes of conformation plots for sphere-like polysaccharides in thermodynamically solvents are $<0.3$. The conformation plot slopes of A130-20 and SH1-20 were 0 and 0.06, respectively, indicating both A130-20 and SH1-20 were solid sphere-like structure. However, the slopes of A130-20 was smaller than that of SH1-20, which provide additional evidence of branching after mutation.
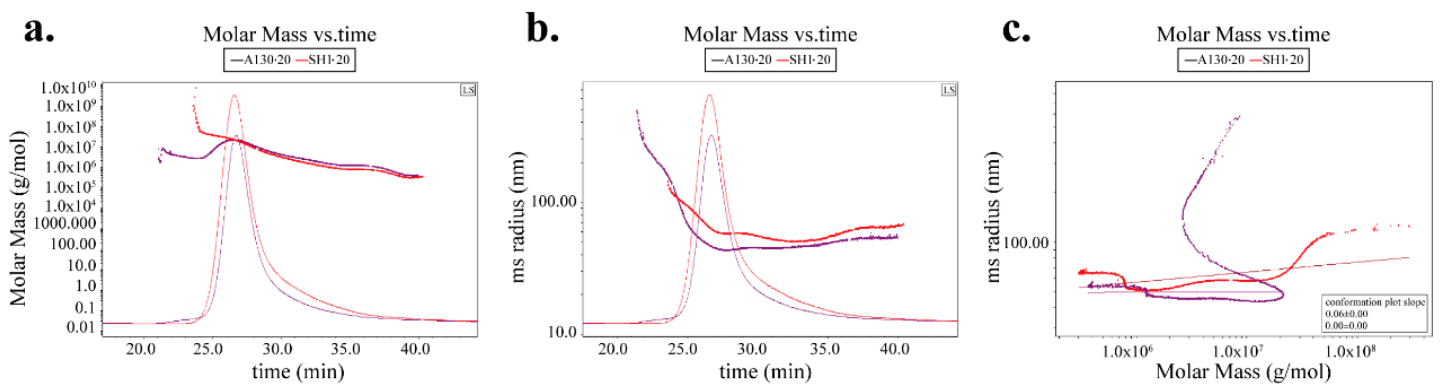

Figure 3. The polysaccharide conformation analysis of A130-20 and SH1-20. (a) Molar mass vs. time chromatogram of A130-20 and SH1-20; (b) rms radius vs. time chromatogram of A130-20 and SH1-20; (c) rms conformation plot of A130-20 and SH1-20.

\subsection{Effects of Mutation on NO Production from RAW 264.7 Cells}

To determine whether S. sanghuang polysaccharides were detrimental to RAW 264.7 cells, the cell viability was examined using the cell counting kit-8 following treatment with different concentrations 
(50, 200, and $500 \mu \mathrm{g} / \mathrm{mL})$ for $24 \mathrm{~h}$. As shown in Figure $4 \mathrm{a}$, six polysaccharide fraction treatment did not induce RAW 264.7 cell death at concentrations up to $500 \mu \mathrm{g} / \mathrm{mL}$.
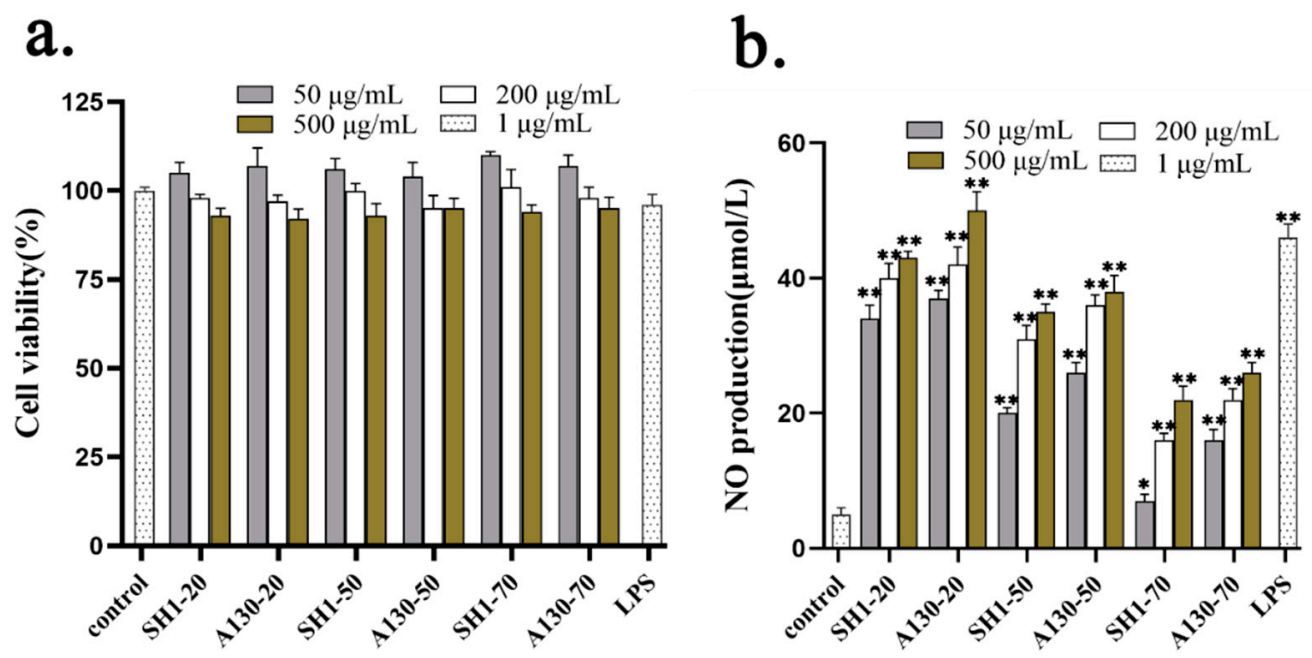

Figure 4. In vitro macrophage activation activities of $S$. sanghuang polysaccharides. (a) cell cytotoxicity test of six polysaccharide fractions; (b) The effects of six polysaccharide fractions on NO Production from RAW 264.7 cells. Each value represents the mean \pm SD. ${ }^{*} p<0.05,{ }^{* *} p<0.01$ compared to control group.

$\mathrm{NO}$ is a non-specific effector molecule synthesized by nitric oxide synthase (NOS), and it plays a key role in inhibiting the growth of various pathogenic microorganisms and in the regulation of tumor cell apoptosis [22,23]. As shown in Figure 4b, SH1-20 and A130-20 significantly stimulated the NO release of murine macrophage. NO content increased markedly in the presence of A130-20 ranging from 50 to $500 \mu \mathrm{g} / \mathrm{mL}$, which was higher than that of SH1-20. This indicated ARTP mutation promoted the macrophage activation activities by producing new macromolecule polysaccharides. A130-50 and SH1-50 also have certain effects on improving NO production, and A130-50 showed better in vitro macrophage activation activities than SH1-50. The macrophage activation activities reduced with the molecular weight of polysaccharides decreased. The results revealed that the large-molecular-weight polysaccharides were the main components contributing to immune activity in vitro [22]. The immunity actives of polysaccharides isolated from A130 improved might related to the production of new macromolecular polysaccharide by ARTP mutagenesis and the change of chain conformation and molecular weight distribution.

\subsection{Effects of Mutation on Immunostimulatory Activity}

Proinflammatory cytokines such as IL-6, IL-1 $\beta$ and TNF- $\alpha$ play important pathological effects in various diseases and regulation of immune function [24]. The effects on cellular release of inflammatory cytokines IL-6, TNF- $\alpha$ and IL-1 $\beta$ induced by polysaccharide fractions from submerged fermentation mycelia of SH1 and A130 were shown in Figure 5.

The cytokine release of IL- 6 , IL- $1 \beta$ and TNF- $\alpha$ was elevated by $S$. sanghuang polysaccharide fractions in a significant dose-dependent manner by ELISA test. In Figure 5a, compared with A130-70 and SH1-70 groups, A130-20, SH1-20, and A130-50, SH1-50 significantly increased the cytokine release of IL- 6 at $500 \mu \mathrm{g} / \mathrm{mL}$. The A130-20 (50 $\mu \mathrm{g} / \mathrm{mL}$ and $200 \mu \mathrm{g} / \mathrm{mL})$ groups stimulated IL-6 production much higher than that of SH1-20 groups. Meanwhile, A130-20 and SH1-20 groups significantly increased TNF- $\alpha$ production better than A130-50, SH1-50 and A130-70, SH1-70 groups at concentrations of 50, 200 , and $500 \mu \mathrm{g} / \mathrm{mL}$, respectively (Figure $5 \mathrm{~b}$ ). These indicated that macromolecular polysaccharides (20\% ethanol-precipitated fractions) showed better stimulating activities on IL- 6 and TNF- $\alpha$ production, and polysaccharide fractions extracted from mutagenic strain A130 mycelia showed higher stimulating 
activities on IL-6 and TNF- $\alpha$ production compared with original strain SH1. Moreover, A130-20 clearly increased IL-1 $\beta$ production higher than the A130-50 and A130-70 groups at concentrations of 50, 200, and $500 \mu \mathrm{g} / \mathrm{mL}$, respectively, and similar as SH1 group (Figure 5c). A130-20 had better stimulating activity on IL-1 $\beta$ release at $50 \mu \mathrm{g} / \mathrm{mL}$ than SH1-20, as shown in Figure $5 \mathrm{c}$. The results showed the enhanced immunological activity in vitro was correlated with the cytokine expression of IL-6, TNF- $\alpha$ and IL-1 $\beta$. They also indicated that macromolecular polysaccharide fractions from mycelia of $S$. sanghuang functions importantly on their biological activities, and large molecular weight polysaccharide fractions derived from $S$. sanghuang mutagenic strain promoted proinflammatory cytokine production $[25,26]$.
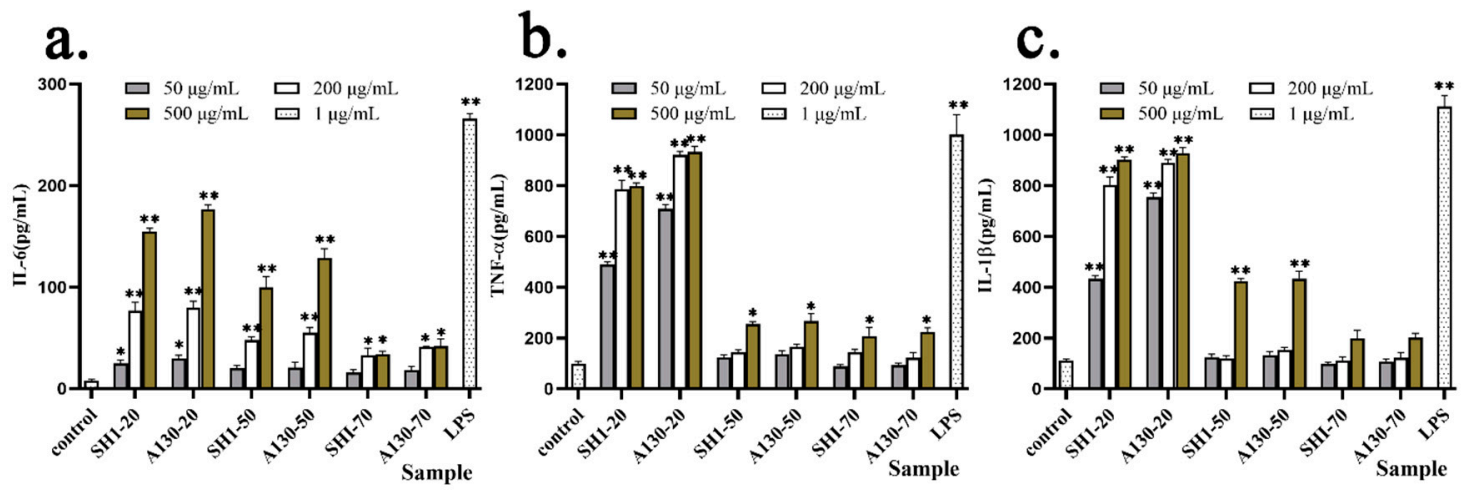

Figure 5. Effect of six polysaccharide factions on cytokine release of IL-6 (a), TNF- $\alpha$ (b) and IL-1 $\beta$ (c) from THP-1cells. Each value represents the mean $\pm \mathrm{SD}$. ${ }^{*} p<0.05,{ }^{* *} p<0.01$ compared to the negative control.

\section{Materials and Methods}

\subsection{Strains and Cells}

The original strain SH1 was obtained from China's edible fungi sub-center of microbial culture collection center. The mutant strain A130 were bred by automated ARTP mutation breeding system and screened. After the ARTP treatment and screening, the optimal strain A130 was identified with genetic stability in the submerged culture experiment.

Murine macrophage cell line RAW264.7 cells and human monocytic cell line THP-1 cells were purchased from the Type Culture Collection of the Chinese Academy of Sciences.

\subsection{Submerged Culture}

Both original strain and mutant strains were preserved in slant medium containing $(\mathrm{g} / \mathrm{L})$ : Potato Dextrose Agar (BD Difco, Sparks, MD, America) 39.0, mulberry powder 10.0; The seed culture was conducted in a $250 \mathrm{~mL}$ flask for 7 days at $28{ }^{\circ} \mathrm{C}$ containing $100 \mathrm{~mL}$ liquid medium $(\mathrm{g} / \mathrm{L})$ : Potato Dextrose Broth (BD Difco, America) 24.0, mulberry powder 10.0; Fermentation medium (g/L): $\mathrm{KH}_{2} \mathrm{PO}_{4} 1.0, \mathrm{MgSO}_{4} 1.0$, yeast autolytic powder 10.0 and glucose 20.0 and mulberry powder 10.0. The submerged culture was performed with three duplicate parallel samples in $1 \mathrm{~L}$ flask for 5 days with $10 \%(v / v)$ seed culture. The mycelia from three parallel samples were collected and freeze-dried, respectively. The dry weights of three parallel mycelia samples were accurately weighed for determining biomass production and polysaccharide contents.

\subsection{Polysaccharides Extraction and Isolation}

$500 \mathrm{~g}$ mycelia samples were accurately weighed and immersed in $9 \mathrm{~L}$ distilled water overnight before extraction. Subsequent extraction of the mycelia polysaccharides was performed at $100{ }^{\circ} \mathrm{C}$ for $4 \mathrm{~h}$. The extracts were evaporated before being centrifuged to give the supernatant, which was then separated by gradient alcohol precipitation to 20,50 and $70 \%$. The precipitates were dissolved 
in distilled water and dialyzed to remove small molecule substance for three days at $4{ }^{\circ} \mathrm{C}$ with the following freeze-dry to yield polysaccharide fractions, named as SH1-20, SH1-50, SH1-70 and (A130-20, A130-50, A130-70) respectively. The protocols of polysaccharide extraction process were shown in Figure 6.

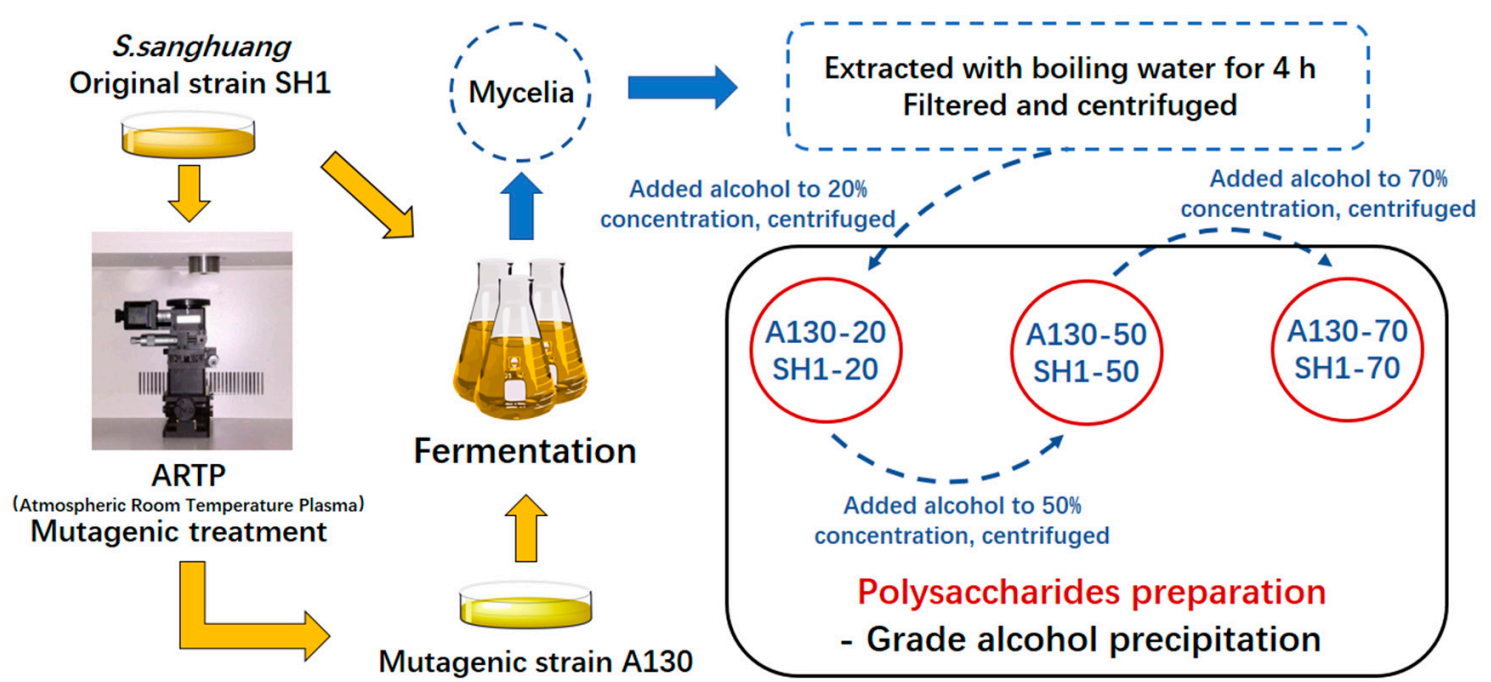

Figure 6. The protocol of polysaccharide extraction process.

\subsection{Physicochemical Characterization of S. sanghuang Polysaccharides}

\subsubsection{Mycelial Biomass and Polysaccharide Content of Different S. sanghuang Strains}

Dry weight of mycelia was measured to calculate the biomass. The total polysaccharide contents of $S$. sanghuang mycelia were calculated by the phenol-sulfuric acid method.

\subsubsection{Monosaccharide Analysis}

High-performance anion exchange chromatography (HPAEC) (Dionex, Sunnyvale, CA, USA) was used to determine the monosaccharide composition of six different polysaccharide fractions according to the literature with little modification [27]. $2 \mathrm{mg}$ samples were hydrolyzed at $110{ }^{\circ} \mathrm{C}$ for $4 \mathrm{~h}$ with $3 \mathrm{~mL}$ $2 \mathrm{moL} / \mathrm{L}$ trifluoroacetic acid (TFA) in a vial container. The hydrolysate was heated and evaporated with methanol by $\mathrm{N}_{2}$ blowing to completely remove TFA. Then the hydrolysates were dissolved by deionized water and filtered. $25 \mu \mathrm{L}$ hydrolyzed sample was injected into HPAEC equipped with a CarboPac ${ }^{\mathrm{TM}} \mathrm{PA} 20$ column (Thermo, Sunnyvale, CA, USA). The samples were eluted with mobile phase at $30^{\circ} \mathrm{C}$, and separate by monosaccharide standards [28].

\subsubsection{HPSEC-MALLS-UV-VIS-RI Measurements}

High performance size-exclusion chromatography combined with multi-angle laser light scattering (HPSEC-MALLS) is often used to determine distributions of molecular weight, size and composition independent of column calibration by reference standards [29]. The measurements were conducted on eight-angle laser photometer (Wyatt Technology Co., Santa Barbara, CA, USA) equipped with TSK-GEL G6000 and G4000 PWXL column in $0.5 \mathrm{M} \mathrm{NaCl}$ aqueous solution at $38^{\circ} \mathrm{C}$. A differential refractive index detector (RI-2414, Waters, Milford, MI, USA), and ultraviolet detector (UV-2487, Waters, Milford, MI, USA) were simultaneously connected. Meanwhile, an online differential viscometer (ViscoStar ${ }^{\mathrm{TM} I I}$, Wyatt Technology Co., Goleta, CA, USA) was used to determine the intrinsic viscosity of polysaccharides [30]. All solutions with a concentration of $5 \mathrm{mg} / \mathrm{mL}$ were first centrifuged for $15 \mathrm{~min}$ with at $12,000 \times g$, followed by $0.25 \mu \mathrm{m}$ filter and injected onto the HPLC system. The dn/dc values of samples in aqueous $0.5 \mathrm{M} \mathrm{NaCl}$ were determined to be $0.142 \mathrm{~mL} / \mathrm{g}$. Huggins and Kraemer plots 
were used to estimate the intrinsic viscosity [ๆ]. ASTRA 6.1 software (Wyatt Technology, Santa Barbara, CA, USA) was utilized for the data acquisition and analysis [31].

\subsubsection{Fourier Transform Infrared Spectrum Analysis of Polysaccharide Fractions}

An amount of $2 \mathrm{mg}$ polysaccharide samples were ground and uniformly mixed with $\mathrm{KBr}$ powder, and infrared spectrum was recorded ranging from $4000-400 \mathrm{~cm}^{-1}$ after tablet compressing by Nicolet FT-IR spectrometer (Thermo, Waltham, MA, USA).

\subsection{Assay of the Immunological Activity}

\subsubsection{Cell Culture and Cell Viability Measurements}

RAW 264.7 cells was cultured in Dulbecco's Modified Eagle's medium (DMEM) containing 10\% heat-inactivated FBS and 1\% antibiotics.

Cell viability was determined by CCK-8 assay kit to explore the cytotoxicity of S. sanghuang polysaccharides on RAW 264.7 cells. Aliquots of RAW 264.7 cell suspension $\left(180 \mu \mathrm{L} ; 5 \times 10^{5}\right.$ cells $\left./ \mathrm{mL}\right)$ with $20 \mu \mathrm{L}$ different concentrations of polysaccharide samples were incubated at $37{ }^{\circ} \mathrm{C}$ in $5 \% \mathrm{CO}_{2}$ for $24 \mathrm{~h}$. Phosphate buffered saline (PBS) was served as the negative control. The cell viability rate was calculated according to protocol [32].

\subsubsection{Determination of NO Production}

Here, $5 \times 10^{5}$ cells/well RAW 264.7 macrophage cells were cultured in a 96-well plate; six polysaccharide fractions with various final concentrations $(50,200$, and $500 \mu \mathrm{g} / \mathrm{mL})$ were added to the cells for $48 \mathrm{~h}$. Nitrite contents were determined through the Griess reaction. $1 \mu \mathrm{g} / \mathrm{mL} \mathrm{PBS}$ and lipopolysaccharides (LPS) served as negative and positive controls, respectively.

\subsubsection{THP-1 Macrophage Differentiation and Quantitative Cytokines Trial}

THP-1 macrophage differentiation and quantitative cytokines trial were performed according to literature with little modification [33].

The six polysaccharide fractions were dissolved with sterilized PBS solution and diluted to final concentration of 50,200 , and $500 \mu \mathrm{g} / \mathrm{mL}$. THP-1 cells were differentiated to form a mature macrophage-like state by phorbol 12-myristate 13-acetate (PMA, Sigma-Aldrich). After treatment with PMA for $48 \mathrm{~h}$, the medium was removed, and fresh medium $(180 \mu \mathrm{L})$ and serial concentrations of samples $(20 \mu \mathrm{L})$ were added into 96-well plate. The cell supernatants were collected after being co-cultured for $48 \mathrm{~h}$. The cytokine levels (pg/mL) of culture supernatant were determined by human IL-6, IL-1 $\beta$ and TNF- $\alpha$ ELISA kit according to the manufacturer's instructions, respectively. All data were expressed as mean \pm SD and analyzed by one-way analysis of variance (ANOVA).

\section{Conclusions}

S. sanghuang is a rare medicinal fungus has been attracted more and more attention due to its remarkable anti-tumor and immunomodulatory effects. The structure and bioactivities of polysaccharides from $S$. sanghuang have been reported and proven to be main functional components [34]. The increase of polysaccharide content will be of great value for S. sanghuang development and utilization. A new strain, A130, screened from the mutant strains by ARTP mutagenesis, with higher polysaccharide content in submerged fermentation mycelia, was obtained in our previous study and the intracellular polysaccharide yield of A130 was improved to 1.2-fold of that of the wild strain SH1. The structural characteristics and biological activities of intracellular polysaccharide derived from mutagenic $S$. sanghuang strain were investigated in this study. The polysaccharides differ in chemical composition, molecular mass, chain conformation and immune activity in vitro after mutation, and the main differences were found in polysaccharide fractions precipitated by $20 \%$ ethanol 
which showed a relatively expanded branching chain with higher molecular weight and better in vitro macrophage activation activities after mutation.

The study also showed that ARTP was a novel, powerful, and environmentally friendly mutagenesis tool for generating high-polysaccharide-yield S. sanghuang strain, and may therefore contribute to the large-scale utilization of the rare medicinal fungi. However, much more extensive research, as well as technological development, has to be carried out in future work. The mechanism of high polysaccharide production by ATRP mutation, and the key enzymes and genes in polysaccharide synthetic metabolic pathways need to be discovered. In addition, a basic understanding of both the primary and secondary structures of polysaccharides produced by ARTP need to be further studied to better elucidate the structure-activity relationship.

Author Contributions: T.L. wrote the original manuscript. Y.Y. and W.W. designed the experiments and revised the manuscript; L.C. performed the experiments and data curation; and D.W., G.D., W.C. and H.Z. collected the data. All authors have read and agreed to the published version of the manuscript.

Funding: This research was funded by Shanghai “Chenguang Program” (Grant No. 14CGB21) and Shanghai's leading talents program.

Acknowledgments: Shanghai “Chenguang Program” and Shanghai's leading talents program.

Conflicts of Interest: The authors declare no conflict of interest.

\section{References}

1. Zhou, L.; Ghobadnejhad, M.; Tian, X.; Wang, Y.; Wu, F. Current Status of 'Sanghuang' as a Group of Medicinal Mushrooms and Their Perspective in Industry Development. Food Rev. Int. 2020, 1-19. [CrossRef]

2. Chen, H.; Tian, T.; Miao, H.; Zhao, Y.Y. Traditional uses, fermentation, phytochemistry and pharmacology of Phellinus linteus: A review. Fitoterapia 2016, 113, 6-26. [CrossRef]

3. Wen, Y.; Wan, Y.Z.; Qiao, C.X.; Xu, X.F.; Wang, J.; Shen, Y. Immunoregenerative effects of the bionically cultured Sanghuang mushrooms (Inonotus sanghuagn) on the immunodeficient mice. J. Ethnopharmacol. 2019, 245, 112047. [CrossRef] [PubMed]

4. $\quad$ Lin, W.C.; Deng, J.S.; Huang, S.S.; Wu, S.H.; Chen, C.C.; Lin, W.R.; Lin, H.Y.; Huang, G.J. Anti-Inflammatory Activity of Sanghuangporus sanghuang Mycelium. Int. J. Mol. Sci. 2017, 18, 347. [CrossRef]

5. Lin, W.C.; Deng, J.S.; Huang, S.S.; Wu, S.H.; Lin, H.Y.; Huang, G.J. Evaluation of antioxidant, anti-inflammatory and anti-proliferative activities of ethanol extracts from different varieties of Sanghuang species. RSC Adv. 2017, 7, 7780-7788. [CrossRef]

6. Ma, X.K.; She, X.; Peterson, E.C.; Wang, Y.Z.; Zheng, P.; Ma, H.; Zhang, K.; Liang, J. A newly characterized exopolysaccharide from Sanghuangporus sanghuang. J. Microbiol. 2019, 57, 812-820. [CrossRef]

7. Zhang, X.; Zhang, X.F.; Li, H.P.; Wang, L.Y.; Zhang, C.; Xing, X.H.; Bao, C.Y. Atmospheric and room temperature plasma (ARTP) as a new powerful mutagenesis tool. Appl. Microbiol. Biotechnol. 2014, 98, 5387-5396. [CrossRef]

8. Yuan, L.; Wang, L.; Ma, K.; Guo, L.; Chong, Z.; Zhao, H.; Lai, Q.; Li, H.P.; Xing, X.H. Characteristics of hydrogen production of an Enterobacter aerogenes mutant generated by a new atmospheric and room temperature plasma (ARTP). Biochem. Eng. J. 2011, 55, 17-22.

9. Wang, L.Y.; Huang, Z.L.; Li, G.; Zhao, H.X.; Xing, X.H.; Sun, W.T.; Li, H.P.; Gou, Z.X.; Bao, C.Y. Novel mutation breeding method for Streptomyces avermitilis using an atmospheric pressure glow discharge plasma. J. Appl. Microbiol. 2010, 108, 851-858. [CrossRef]

10. Cao, S.; Zhou, X.; Jin, W.; Wang, F.; Tu, R.; Han, S.; Chen, H.; Chen, C.; Xie, G.J.; Ma, F. Improving of lipid productivity of the oleaginous microalgae Chlorella pyrenoidosa via atmospheric and room temperature plasma (ARTP). Bioresour. Technol. 2017, 244, 1400-1406. [CrossRef] [PubMed]

11. Jin, L.; Fang, M.; Zhang, C.; Jiang, P.; Bao, C. Operating conditions for the rapid mutation of the oleaginous yeast by atmospheric and room temperature plasmas and the characteristics of the mutants. Chin. J. Biotechnol. 2011, 27, 461-467.

12. Xu, F.; Wang, J.; Chen, S.; Qin, W.; Yu, Z.; Zhao, H.; Xing, X.; Li, H. Strain improvement for enhanced production of cellulase in Trichoderma viride. Prikl. Biokhim. Mikrobiol. 2011, 47, 61-65. [CrossRef] 
13. Jiang, W.; Zhang, Q.Q.; Tang, W.J.; Cheng, Y.Y.; University, A.P. Research on screening and breeding of Monascus on the production of extracellular polysaccharides by UV-ARTP composite mutagenesis. Food Ferment. Ind. 2016, 42, 64-69.

14. Fang, M.; Jin, L.; Chong, Z.; Tan, Y.; Jiang, P.; Nan, G.; Li, H.; Xing, X.; Joy, S. Rapid Mutation of Spirulina platensis by a New Mutagenesis System of Atmospheric and Room Temperature Plasmas (ARTP) and Generation of a Mutant Library with Diverse Phenotypes. PLoS ONE 2013, 8, e77046C. [CrossRef] [PubMed]

15. Wei, D.; Huang, Q.; He, H. Effect of Atmospheric Pressure Room-temperature Plasma on Mycelia of Ganoderma Lingzhi. J. Anhui Sci. Technol. Univ. 2020, 34, 32-36. (In Chinese)

16. Zhu, L.; Wu, D.; Zhang, H.; Li, Q.; Zhang, Z.; Liu, Y.; Zhou, S.; Wang, W.; Li, Z.; Yang, Y. Effects of Atmospheric and Room Temperature Plasma (ARTP) Mutagenesis on Physicochemical Characteristics and Immune Activity In Vitro of Hericium erinaceus Polysaccharides. Molecules 2019, 24, 262. [CrossRef]

17. Wyatt, P.J. Measuring nanoparticles in the size range to $2000 \mathrm{~nm}$. J. Nanopart. Res. 2018, 20, 322. [CrossRef]

18. Liu, Y.; Tang, Q.; Zhang, J.; Xia, Y.; Yang, Y.; Wu, D.; Fan, H.; Cui, S.W. Triple helix conformation of beta-d-glucan from Ganoderma lucidum and effect of molecular weight on its immunostimulatory activity. Int. J. Biol. Macromol. 2018, 114, 1064-1070. [CrossRef]

19. Cui, W.; Wood, P.J.; Blackwell, B.; Nikiforuk, J. Physicochemical properties and structural characterization by two-dimensional NMR spectroscopy of wheat $\beta$-D-glucan-comparison with other cereal $\beta$-D-glucans. Carbohydr. Polym. 2000, 41, 249-258. [CrossRef]

20. Podzimek, S.; Hermannova, M.; Bilerova, H.; Bezakova, Z.; Velebny, V. Solution properties of hyaluronic acid and comparison of SEC-MALS-VIS data with off-line capillary viscometry. J. Appl. Polym. Sci. 2010, 116, 3013-3020. [CrossRef]

21. Hu, T.; Huang, Q.; Wong, K.; Yang, H. Structure, molecular conformation, and immunomodulatory activity of four polysaccharide fractions from Lignosus rhinocerotis sclerotia. Int. J. Biol. Macromol. 2016, 94, 423-430. [CrossRef] [PubMed]

22. Bai, Y.; Jiang, Y.; Liu, T.; Li, F.; Zhang, J.; Luo, Y.; Zhang, L.; Yan, G.; Feng, Z.; Li, X.; et al. Xinjiang herbal tea exerts immunomodulatory activity via TLR2/4-mediated MAPK signaling pathways in RAW264.7 cells and prevents cyclophosphamide-induced immunosuppression in mice. J. Ethnopharmacol. 2019, 228, 179-187. [CrossRef] [PubMed]

23. Liu, Y.; Zhang, J.; Tang, Q.; Yang, Y.; Guo, Q.; Wang, Q.; Wu, D.; Cui, S.W. Physicochemical characterization of a high molecular weight bioactive beta-D-glucan from the fruiting bodies of Ganoderma lucidum. Carbohydr. Polym. 2014, 101, 968-974. [CrossRef] [PubMed]

24. Jung, G.H.; Kang, J.H. Efficacy of Phellinus linteus (sanghuang) extract for improving immune functions: Study protocol for a randomized, double-blinded, placebo-controlled pilot trial. Medicine 2020, 99, e18829. [CrossRef]

25. Chan, G.C.; Chan, W.K.; Sze, D.M. The effects of beta-glucan on human immune and cancer cells. J. Hematol. Oncol. 2009, 2, 25. [CrossRef]

26. Akramiene, D.; Kondrotas, A.; Didziapetriene, J.; Kevelaitis, E. Effects of beta-glucans on the immune system. Medicina 2007, 43, 597-606. [CrossRef]

27. Comparison of the preliminary characterizations and antioxidant properties of polysaccharides obtained from Phellinus baumii growth on different culture substrates. Carbohydr. Polym. 2015, 132, 397-399. [CrossRef]

28. Li, T.; Yang, Y.; Liu, Y.; Zhou, S.; Yan, M.Q.; Wu, D.; Zhang, J.; Tang, C. Physicochemical characteristics and biological activities of polysaccharide fractions from Phellinus baumii cultured with different methods. Int. J. Biol. Macromol. 2015, 81, 1082-1088. [CrossRef]

29. Tao, Y.; Zhang, L. Characterization of polysaccharide-protein complexes by size-exclusion chromatography combined with three detectors. Carbohydr. Res. 2008, 343, 2251-2257. [CrossRef]

30. Li, J.; Gu, F.; Cai, C.; Hu, M.; Fan, L.; Hao, J.; Yu, G. Purification, structural characterization, and immunomodulatory activity of the polysaccharides from Ganoderma lucidum. Int. J. Biol. Macromol. 2020, 143, 806-813. [CrossRef]

31. Jin, Y.; Zhang, L.; Chen, L.; Chen, Y.; Cheung, P.C.; Chen, L. Effect of culture media on the chemical and physical characteristics of polysaccharides isolated from Poria cocos mycelia. Carbohydr. Res. 2003, 338, 1507-1515. [CrossRef]

32. Pandey, M.K.; Sung, B.; Ahn, K.S.; Kunnumakkara, A.B.; Chaturvedi, M.M.; Aggarwal, B.B. Gambogic acid, a novel ligand for transferrin receptor, potentiates TNF-induced apoptosis through modulation of the nuclear factor-kappaB signaling pathway. Blood 2007, 110, 3517-3525. [CrossRef] [PubMed] 
33. Daigneault, M.; Preston, J.A.; Marriott, H.M.; Whyte, M.K.; Dockrell, D.H. The identification of markers of macrophage differentiation in PMA-stimulated THP-1 cells and monocyte-derived macrophages. PLoS ONE 2010, 5, e8668. [CrossRef] [PubMed]

34. Synytsya, A.; Novak, M. Structural diversity of fungal glucans. Carbohydr. Polym. 2013, 92, 792-809. [CrossRef] [PubMed]

Sample Availability: Samples of the compounds are not available from authors.

(C) 2020 by the authors. Licensee MDPI, Basel, Switzerland. This article is an open access article distributed under the terms and conditions of the Creative Commons Attribution (CC BY) license (http://creativecommons.org/licenses/by/4.0/). 